\title{
LISTE DES AUTEURS
}

Dominique BARTHÉLEMY, professeur à l'Université de Paris IV, directeur d'études à la IV $V^{e}$ section de l'École pratique des hautes études

Jens Peter CLAUSEN, Universität Bonn

Jean DufouR, directeur d'études à la $\mathrm{IV}^{\mathrm{e}}$ section de l'École pratique des hautes études

Dr. Julian FüHRER, Universität Jena

Françoise GASPARRI, directeur de recherche au CNRS

Dr Lindy GRANT, Courtauld Institute of Art, London

Priv.-Doz. Dr. Rolf GRossE, Institut historique allemand de Paris

Olivier GUYOTJEANNIN, professeur à l'École nationale des chartes

Priv.-Doz. Dr. Frank G. HIRSCHMANN, Universität Trier

Laurent MORELLE, directeur d'études à la IV $\mathrm{IV}^{\mathfrak{e}}$ section de l'École pratique des hautes études

Prof. Dr. Andreas SPEER, Universität Würzburg

Michaël WYsS, archéologue municipal, Ville de Saint-Denis 